\title{
Avaliação da qualidade de vida dos pacientes com insuficiência cardíaca
}

\author{
Assessment of the quality of life of patients with heart failure \\ Evaluación de la calidad de vida de pacientes con insuficiencia cardíaca
}

Natália Mendes Aguiar ${ }^{1 *}$, Paulo Sergio Leahy Andrade Junior ${ }^{1}$, Camila Santana Silva ${ }^{1}$, Iris Lorena Fernandes Santos ${ }^{1}$, Diovanna Lima Silva ${ }^{1}$, João Vitor Rodrigues Freitas ${ }^{1}$, Ana Corina Bandeira Almeida Rodrigues de Oliveira ${ }^{1}$, Luisa Nery Matos ${ }^{1}$, Paloma Monayza Miranda Cardoso ${ }^{1}$, Shesllen Mikaelly Cruz Corrêa'1.

\section{RESUMO}

Objetivo: Avaliar a qualidade de vida dos pacientes com insuficiência cardíaca com fração de ejeção reduzida cadastrados no protocolo de uso do metoprolol em um município do interior da Bahia. Métodos: Trata-se de um estudo quantitativo, descritivo e transversal com nível da pesquisa exploratório. A pesquisa foi realizada em entrevista com Usuários cadastrados no Protocolo de Uso do Metoprolol, vinculado às Farmácias da Família de um município do interior da Bahia e posteriormente foi aplicado o questionário MLHFQ. Os dados obtidos foram tabelados e analisados em planilhas do Excel® versão 2013. O estudo foi aprovado por Comitê de Ética em Pesquisa. Resultados: A amostra foi de 34 participantes, em sua maioria do gênero masculino, com média de idade de 69,73 anos, semelhante aos outros estudos e, além disso, foi observado que a IC representa uma das causas mais comuns de incapacidade, em concordância com a maioria das pesquisas. Conclusão: Foi observado que a maioria dos participantes possuíam moderada qualidade de vida, o que não está totalmente relacionado ao declínio da Fração de Ejeção do Ventrículo Esquerdo (FEVE).

Palavras-chave: Envelhecimento populacional, Insuficiência cardíaca, Metoprolol, Qualidade de vida.

\begin{abstract}
Objective: To evaluate the quality of life of patients with heart failure with reduced ejection fraction registered in the protocol for the use of metoprolol in a city in the interior of Bahia. Methods: This is a quantitative, descriptive and cross-sectional study with an exploratory research level. The research was conducted in interviews with users registered in the Protocol for the Use of Metoprolol, linked to Family Pharmacies in a municipality in the interior of Bahia, and the MLHFQ questionnaire was subsequently applied. The data obtained were tabulated and analyzed in Excel® version 2013 spreadsheets. The study was approved by the Research Ethics Committee. Results: The sample consisted of 34 participants, mostly male, with a mean age of 69.73 years, similar to other studies and, in addition, it was observed that HF represents one of the most common causes of disability in agreement with most research. Conclusion: It was observed that most participants had moderate quality of life, which is not entirely related to the decline in Left Ventricular Ejection Fraction (LVEF).
\end{abstract}

Key words: Population aging, Heart failure, Metoprolol, Quality of life.

\section{RESUMEN}

Objetivo: Evaluar la calidad de vida de pacientes con insuficiencia cardíaca con fracción de eyección reducida registrados en el protocolo de uso de metoprolol en una ciudad del interior de Bahía. Métodos: Se trata de un estudio cuantitativo, descriptivo y transversal con un nivel de investigación exploratorio. La investigación se realizó en entrevistas a usuarios registrados en el Protocolo de Uso de Metoprolol, vinculado a Farmacias

1 Faculdade Santo Agostinho (FASA), Vitória da Conquista - BA. *E-mail: natycte@icloud.com SUBMETIDO EM: 7/2021 
Familiares en una ciudad del interior de Bahía, y posteriormente se aplicó el cuestionario MLHFQ. Los datos obtenidos fueron tabulados y analizados en planillas Excel® versión 2013. El estudio fue aprobado por el Comité de Ética en Investigación. Resultados: La muestra estuvo conformada por 34 participantes, en su mayoría hombres, con una edad media de 69,73 años, similar a otros estudios y, además, se observó que la IC representa una de las causas más comunes de discapacidad de acuerdo con la mayoría de las encuestas. Conclusión: se observó que la mayoría de los participantes presentaba una calidad de vida moderada, lo que no está enteramente relacionado con la disminución de la fracción de eyección del ventrículo izquierdo (FEVI).

Palabras clave: Envejecimiento poblacional, Insuficiencia cardíaca, Metoprolol, Calidad de vida.

\section{INTRODUÇÃO}

O envelhecimento populacional que vem acontecendo nos últimos anos traz consigo um aumento da prevalência de doenças crônicas, dentre elas Insuficiência Cardíaca (IC) que aumenta sua incidência de acordo com o aumento da faixa etária (MAIA RJC, et al., 2019; MURAD RV, et al., 2018; JORGE AJL, et al., 2017). A insuficiência cardíaca é uma comorbidade, na qual o coração não consegue bombear o sangue de forma adequada para atender as demandas metabólicas, ou faz isso as custas de altas pressões de enchimento (MIZZACI CC, et al., 2017).

Esta patologia afeta 6,5 milhões de brasileiros e representa a principal causa de internação hospitalar em pacientes acima de 60 anos, raramente ocorre de forma isolada, estando associada a diversas comorbidades, o que caracteriza um problema de saúde pública com elevados custos ao sistema de saúde (BARBOSA RR, et al., 2014; KUROGI EM, et al., 2020).

A classificação da IC é baseada em diversos parâmetros, dentre eles está o de Fração de Ejeção do Ventrículo Esquerdo (FEVE), que inclui pacientes com FEVE normal ( $\geq 50 \%$ ), ou seja IC com Fração de Ejeção Preservada (ICFEp), e aqueles com FEVE diminuída (<40\%), denominados IC com fração de ejeção reduzida (ICFEr). Há também a classificação funcional da New York Heart Association (NYHA) que considera a gravidade dos sintomas, avaliando a tolerância ao esforço físico. Nesta, os pacientes classe III e IV estão em piores condições clínicas e têm prognóstico mais reservado, já pacientes da classe II encontram-se mais estáveis e possuem menos internações (FERNANDES ADF, et al., 2020).

Este estagiamento da ACC/AHA enfatiza o desenvolvimento da doença, incluindo tanto o estágio A, em que paciente possui fator de risco para desenvolver IC, porém sem doença estrutural; o $B$ no qual os pacientes que já apresentam alteração cardíaca estrutural, mas permanecem assintomáticos; o $C$ inclui aqueles com alteração cardíaca estrutural que desenvolveram sintomas de IC, e por fim, o estágio $D$ em que os pacientes são refratários ao tratamento e requerem intervenção especial. Sendo que uma vez no estágio avançado, o paciente não volta ao anterior, mesmo com o tratamento efetivo, nesta classificação (FREITAS AKE, et al., 2017).

O portador da IC têm sua qualidade de vida prejudicada pela doença, influenciando diretamente a sua rotina, sendo de suma importância sua análise em relação tanto à percepção da doença quanto à maneira como está interfere em suas atividades cotidianas. Para isso, vários instrumentos têm sido utilizados nesses pacientes, sobretudo o Minnesota Living With Heart Failure Questionnaire (MLHFQ), um instrumento específico para a patologia, muito útil, principalmente em idosos (SACCOMANN ICR, et al., 2011; SOUSA MM, et al., 2017; JORGE AJL, et al., 2017).

Visto que a patologia da IC é altamente prevalente, com elevada morbimortalidade e interferência direta na qualidade de vida desses pacientes, estudar a associação dos fatores acima torna o tema com alta relevância na prática médica (SOUSA MM, et al., 2017; LIMA PBD e MORAIS ERD, 2014). Dessa forma, o presente estudo teve como objetivo avaliar a qualidade de vida dos pacientes com insuficiência cardíaca com fração de ejeção reduzida cadastrados no protocolo de uso do metoprolol em um município do interior da Bahia, relacionando-a com os dados existentes na literatura acerca do mesmo. 


\section{MÉTODOS}

Trata-se de um estudo quantitativo, descritivo, transversal com nível da pesquisa exploratório, sendo o conhecimento construído dependente das entrevistas realizadas, como também de informações adquiridas em literatura. A pesquisa foi realizada em entrevista com Usuários cadastrados no Protocolo de Uso do Metoprolol, vinculado às Farmácias da Família de um município do interior da Bahia. Dos 513 usuários cadastrados, foram selecionados para entrevista e avaliação clínica os 158 usuários portadores do CID I50, referente à Insuficiência Cardíaca.

Foram incluídos no estudo: pacientes com Insuficiência Cardíaca, cadastrados no Protocolo de Uso do Metoprolol, vinculado às Farmácias da Família de um município do interior da Bahia, de ambos os sexos e com idade superior à 18 anos. Os critérios de exclusão foram não residir no município de Vitória da Conquista, não estar cadastrado no Protocolo de Uso do Metoprolol, não estar vinculado às Farmácias da Família de um município do interior da Bahia e não estar dentro da faixa etária estipulada, idade inferior a 18 anos.

A pesquisadora contatou os usuários, por telefone, convidando-os para participarem da pesquisa. Os que aceitaram, foram agendados para responderem ao questionário pessoalmente em conformidade com o horário de funcionamento da Farmácia da Família. Trinta e quatro usuários cadastrados aceitaram participar da pesquisa, sendo que estes estavam de acordo com os critérios de inclusão.

Dos 34 pacientes incluídos na pesquisa, 13 entrevistas ocorreram pessoalmente e diante da situação epidemiológica do país, a pandemia do novo Coronavírus, 21 foram entrevistados via telefone em horário comercial e explicando todos os processos da pesquisa, já que estes tinham consentimento do estudo, devido a primeira etapa do estudo que já tinha sido realizada por uma das pesquisadoras.

A coleta de dados foi dividida em etapas, sendo que primeiro foram utilizadas as informações contidas no cadastro do Protocolo de uso do metoprolol e posteriormente foi aplicado o questionário Minnesota Living With Heart Failure Questionnaire (MLHFQ) validado para avaliação qualidade de vida, traduzido para português, contendo dados associando como a IC impediu o paciente de viver como desejava no último mês.

Os pacientes responderam ao questionário que conta com vinte e uma (21) perguntas sendo pontuado de 0 (não) à 5 (demais). Ao final a pontuação foi somada, variando de 0 a 105 pontos, sendo que o paciente com escore mais baixo apresenta uma melhor qualidade de vida. Essas questões reúnem uma dimensão física (de 1 a 7,12 e 13) que estão inter-relacionadas com dispneia e fadiga, uma dimensão emocional (de 17 a 21) e outras questões (de número $8,9,10,11,14,15$ e 16) que, quando somadas às dimensões anteriores, resultam em um escore total. O ponto de corte que classificou o paciente como possuindo uma boa qualidade de vida são os escores de até 26 pontos, moderada 26 - 45 pontos e qualidade de vida ruim acima de 45 pontos (CARVALHO VO, et al., 2009).

Foram analisados os escores do MLHFQ dos pacientes, relacionando com sua idade, Fração de Ejeção, tendo como medicamento base utilizado por todos o Succinato de Metoprolol. Para minimizar ou eliminar os riscos do estudo, relacionados ao constrangimento dos participantes da pesquisa ao exporem suas informações pessoais, as entrevistas foram realizadas em ambiente fechado, apenas com a presença do pesquisador e do participante. Já as que foram realizadas por telefone, também foram em ambiente fechado e sem riscos de gravação de conversa, ou algo relacionado.

Este é um subprojeto intitulado "Avaliação da Qualidade de Vida dos Pacientes com Insuficiência Cardíaca Cadastrados no Protocolo de Uso do Metoprolol em um Município do Interior da Bahia" pertencente à pesquisa "Avaliação do Perfil da Farmacoterapia dos Pacientes com Insuficiência Cadastrados no Protocolo de Uso do Metoprolol em Munícipio do Interior da Bahia" aprovada pelo Polo de Educação Permanente em Saúde da Secretaria Municipal de Vitória da Conquista, e analisado pelo Comitê de Ética em Pesquisa (CEP), sob no CAAE 26368819.3.0000.5578.

Todos os sujeitos participantes da pesquisa assinaram o Termo de Consentimento Livre e Esclarecido (TCLE) e receberam uma cópia deste, assinada também pelo coordenador da pesquisa. Os dados obtidos foram tabelados em planilhas do Excel® versão 2013, a partir do preenchimento do formulário específico para coleta de dados para elaboração dos gráficos e comparação dos resultados. 


\section{RESULTADOS E DISCUSSÃO}

A amostra foi composta por 34 pacientes, sendo 1 excluído por ter interrompido o uso do Succinato de Metoprolol. Assim, 33 (97\%) foram incluídos no estudo, destes, 20 (60,61\%) do sexo feminino e $13(39,39 \%)$ do sexo masculino (Gráfico 1).

Gráfico 1 - Distinção entre os sexos dos participantes da pesquisa. Participantes ( $n=34)$.

\section{Sexo dos participantes}

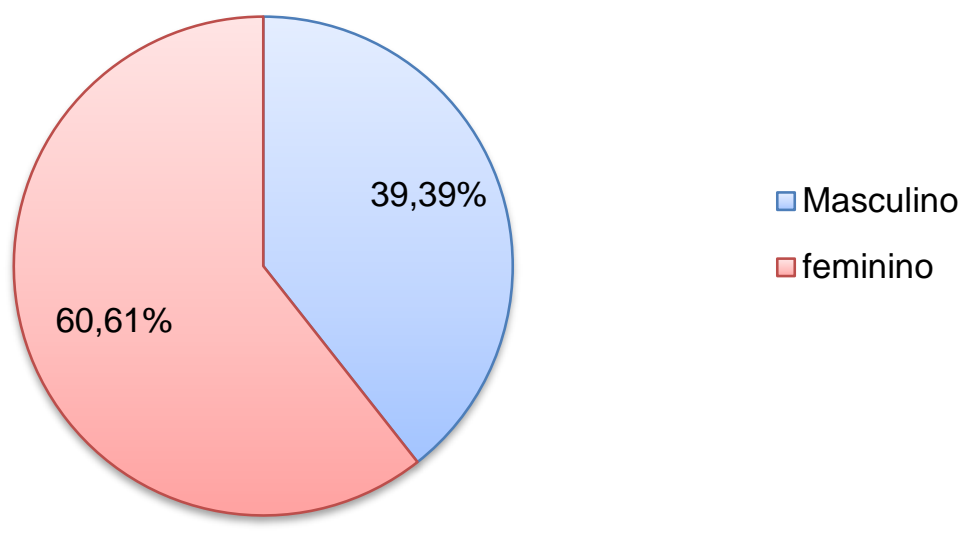

Fonte: Aguiar NM, et al., 2021.

A média de idade calculada entre todos os pacientes foi de 69,73 anos, sendo que $7(21,21 \%)$ não informaram a idade, como consta no Gráfico 2.

Gráfico 2 - Análise da idade da população estudada.

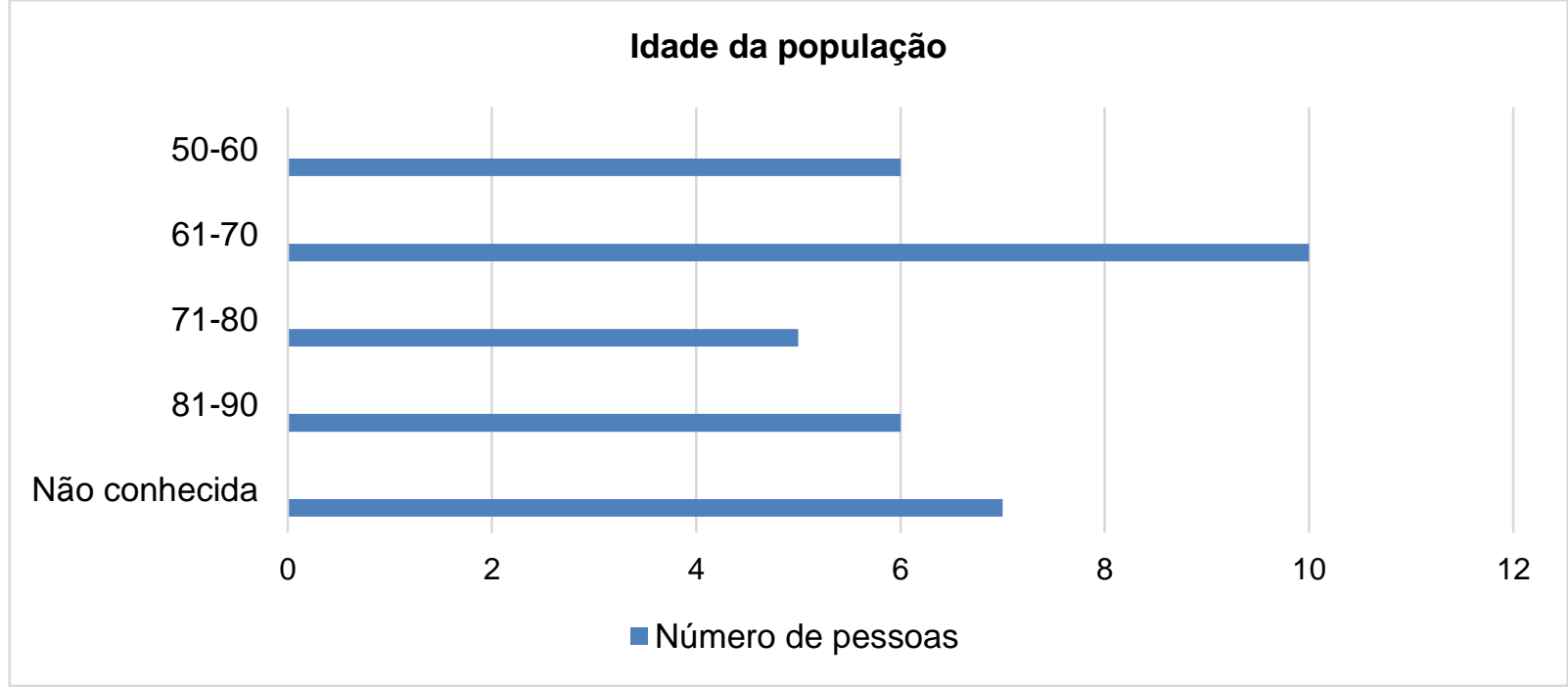

Fonte: Aguiar NM, et al., 2021.

Já 17 (51,51\%) pacientes não informaram a média da fração de ejeção do ventrículo esquerdo (FEVE) ao ecocardiograma por motivos de: não encontrarem o ecocardiograma, não saber interpretar o valor, não ter acesso a este, ou até confundir o ecocardiograma com eletrocardiograma. Portanto, a média da FEVE encontrada na população estudada de acordo com o último ecocardiograma foi $55 \%$. Além disso, 11 pacientes possuem FEVE preservada, 2 FEVE intermediária e 3 FEVE reduzida (Gráfico 3). 
Gráfico 3 - Análise da Fração de Ejeção do Ventrículo Esquerdo (Preservada $\geq 50 \%$, Intermediária 40-49\%, Reduzida $<40 \%$ ou não conhecida) dos pacientes participantes da pesquisa.

\section{Fração de ejeção dos participantes da pesquisa}

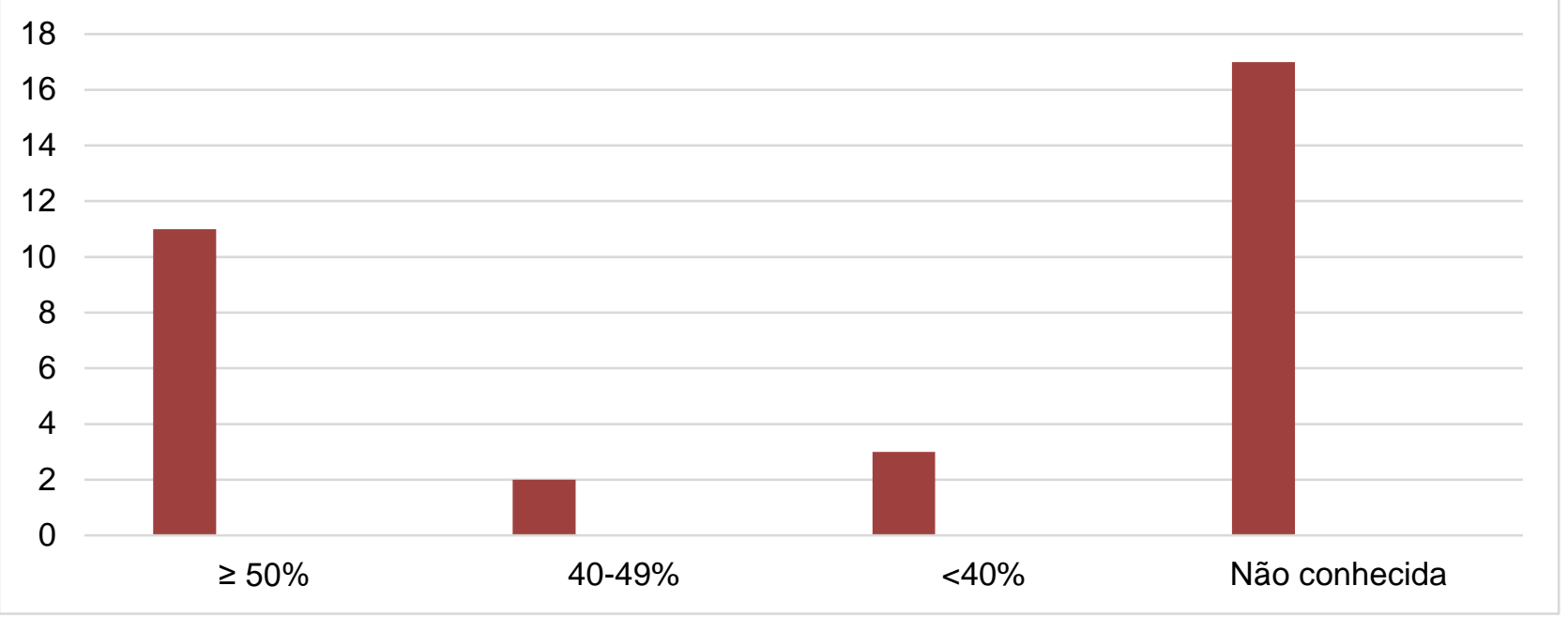

Fonte: Aguiar NM, et al., 2021.

A população entrevistada obteve escores de qualidade de vida bastante diferenciados, de acordo com 0 MLHFQ, e considerando o último mês. Sendo assim, o maior valor entre os participantes foi 83 , enquanto o menor foi 1. A qualidade de vida foi avaliada, sendo 14 participantes, ou seja, $42,42 \%$ possuidores de uma boa qualidade de vida, já que obtiveram pontuação até $26 ; 18,18 \%$ (6 participantes) levam a vida com moderada qualidade, somatória entre 26 e 45, enquanto 13 que representam 40\% possuem uma qualidade de vida ruim, com mais de 45 pontos no MLHFQ. A média dos valores de todos os participantes da pesquisa foi de 34,03 .

Já a comparação entre qualidade de vida e FEVE mostrou que a maioria dos pacientes com FEVE preservada $(\geq 50 \%)$ possuiu qualidade de vida ruim, igual quantidade de pacientes com FEVE intermediária $(40-49 \%)$ mostrou ter qualidade de vida boa e ruim e nenhum moderada. Os participantes com FEVE reduzida $(<40 \%)$, em sua maioria, apresentaram qualidade de vida boa. Dentre os não informantes de FEVE, a maioria também possui qualidade de vida boa (Gráfico 4).

Gráfico 4 - Análise comparativa entre qualidade de vida e FEVE dos participantes da pesquisa, 2021.

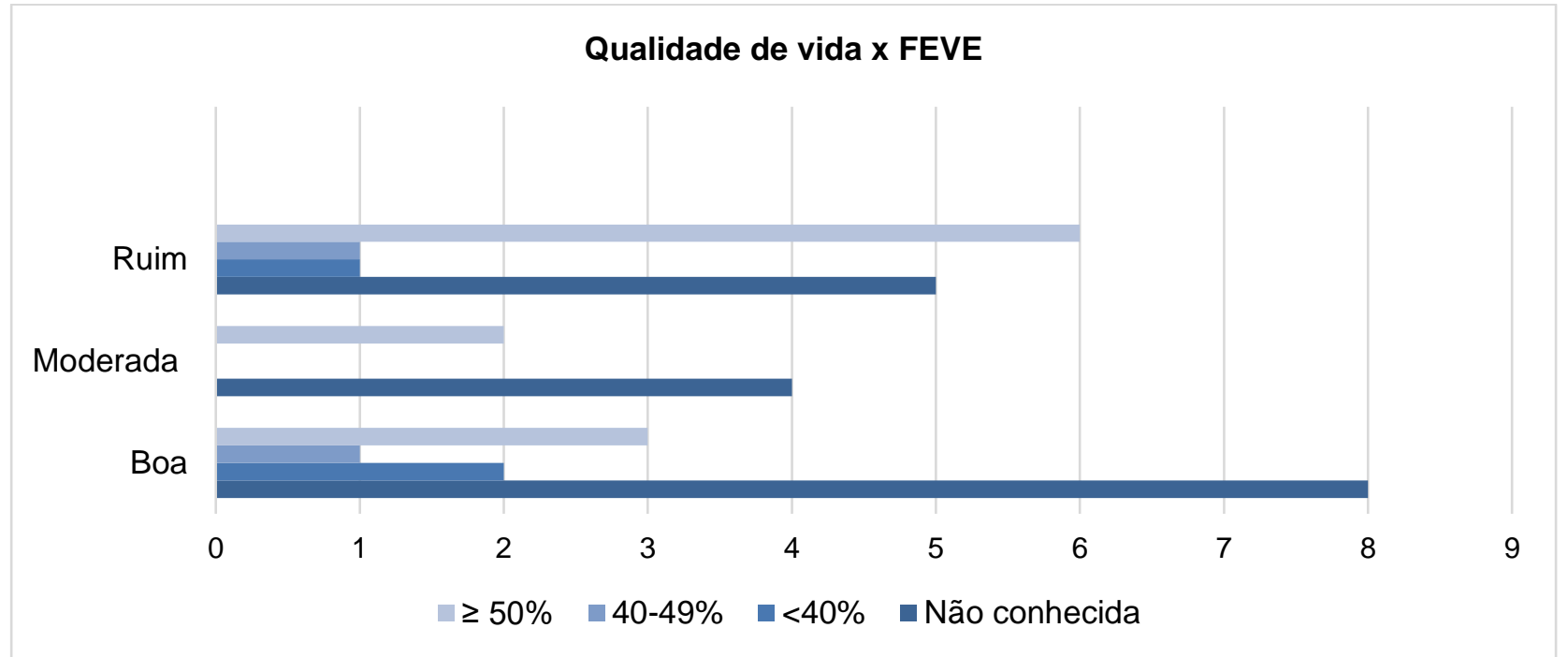

Fonte: Aguiar NM, et al., 2021. 
O presente estudo está em concordância com o fato de que a IC representa uma das causas mais comuns de incapacidade, especialmente, no que se refere à autolimitação da atividade física e restrição das atividades de vida diárias. Além disso, os sintomas causados podem ser acompanhados de perda da memória e dificuldade da capacidade de concentração (KUROGI EM, et al., 2020).

Os portadores da IC têm suas vidas prejudicadas pela doença, influenciando diretamente a rotina do paciente, impondo mudanças ao seu estilo de vida, que inclusive foram citadas pelos pacientes participantes desta pesquisa durante a entrevista realizada e aplicação do questionário (SOUSA MM, et al., 2017; MOURA LZ, et al., 2016). Por isso, o tratamento tanto farmacológico, como não farmacológico da IC, deve buscar reduzir os sintomas, prevenir sua progressão, e reduzir a hospitalização e a mortalidade (MIZZACI CC, et al., 2017).

A qualidade de vida dos pacientes com IC é um complexo objeto de estudo e ainda não está bem estabelecido. Muitos aspectos podem influenciar sua avaliação e estes pontos estimulam sua investigação. A possibilidade de correlacionar a qualidade de vida com a patologia da IC, sua alta morbimortalidade, entre outros fatores, faz com que o tema apresente bastante relevância na prática médica (FIGUEIREDO JHC, et al., 2019).

Existem várias maneiras de qualificar estes pacientes, como vivem e suas limitações, dentre elas, estão os questionários. Este estudo, efetuou a tradução do MLHFQ para a língua portuguesa, estando concordantes, pois não houve dificuldade na aplicação da versão em português do MLHFQ por parte do pesquisador, nem no entendimento do mesmo pelos pacientes envolvidos neste estudo (CARVALHO VO, et al., 2009).

Visto que o elevado perfil etário dos pacientes deste estudo foi semelhante aos desenvolvidos em outros, isso demonstra a importância da avaliação da qualidade de vida dos indivíduos à medida que envelhecem, levando em consideração o que os sujeitos pensam sobre seu estado de saúde (JORGE AJL, et al., 2017). Além disso, o envelhecimento populacional, bem como o aumento da expectativa de vida e a melhoria no tratamento de cardiopatias vem fazendo com que as doenças cardiovasculares aumentem, sendo a IC, a via final de grande parte das cardiopatias e causa frequente de hospitalizações e re-hospitalizações (BOISVERT S, et al., 2015; ROHDE LEP, et al., 2018).

Nesta pesquisa foi verificado que a média dos escores totais dos pacientes estão relacionados a moderada qualidade de vida (26 - 45 pontos), segundo o MLHFQ, estando em conformidade com outros achados, como os de Figueiredo JHC, et al. (2019) que encontraram, a média de escore total do MLHFQ de 37,5; Barbosa RR, et al. (2014) com escore de 40,3; Nogueira IDB, et al. (2010) verificaram pontuações médias de 41,86; Jorge AJL, et al. (2017) encontraram média de 35,3, além de Paz LFA, et al. (2019) que tiveram como resultado 34,3 no questionário.

Já Chiodelli GC, et al. (2015), encontraram um total de 50,3 e Sousa MMD, et al. (2017) de 50,4, provando que os pacientes de ambos os estudos mostram ter uma qualidade de vida ruim, em discordância com o presente estudo. A média de idade nos estudos verificados foi semelhante, englobando em geral, a população em envelhecimento, em concordância com o presente. Sousa MMD, et al. (2017) tiveram como média de idade 77 anos; Jorge AJL, et al. (2017) 67,5 anos e Paz LFA, et al. (2019) 60,3 anos. Apesar de ser considerado idoso a partir de 60 anos de idade, e Barbosa RR, et al. (2014) estudaram um grupo com média de 59,2 anos e Chiodelli GC, et al. (2015) e Nogueira IDB, et al. (2010) encontraram uma média de 52 anos.

Em relação a distribuição de gênero, a atual pesquisa contou com mais mulheres que homens, discordando um pouco dos seguintes estudos, que obtiveram em sua maioria pacientes do sexo masculino. Figueiredo JHC, et al. (2019), contaram com 74\%; Paz LFA, et al. (2019) 57\% e Barbosa RR, et al. (2014) contaram com $78,6 \%$, assim como Sousa MMD, et al. (2017). Que também tiveram a maioria representada por homens (72\%). Porém, em semelhança com os dados desta pesquisa, Nogueira IDB, et al. (2010) e Jorge AJL, et al. (2017) tiveram como maioria o gênero feminino.

Os fatores de risco para IC são: baixo nível educacional, sedentarismo, tabagismo, obesidade, diabetes mellitus, Hipertensão Arterial Sistêmica (HAS), doença valvar do coração, hipertrofia de ventrículo esquerdo, 
doenças coronarianas e sexo masculino, sendo que este último chamou atenção, já que no presente estudo a maioria da população tratava-se do sexo feminino, não tendo como relacionar diretamente a este fator de risco. Porém, vale ressaltar que o atual faz parte de um programa de cadastro de uma população específica (CARVALHO VO, et al., 2009).

Outro ponto importante é que se espera que a FEVE reduzida esteja relacionada a um menor débito cardíaco, e por isso insuficiente em atender a demanda metabólica tissular, esperando sintomas físicos mais severos e, então, piores percepções na qualidade de vida (QV) (SOUSA MMD, et al., 2017). Esta afirmação, no entanto, é controversa, ao passo que alguns autores como Paz LFA, et al. (2019) e Pelegrino VM, et al. (2011), discordam desses achados, assim como ocorreu na presente pesquisa, em que os achados de pior qualidade de vida não estiveram diretamente relacionados com mais baixa FEVE.

\section{CONCLUSÃO}

No presente estudo, que possibilitou a avaliação da QV de pacientes com IC, foi verificado que a maioria apresentou moderada qualidade de vida, e que esta não esteve totalmente relacionada ao declínio da FEVE. Percebeu-se que a análise da QV de pacientes com IC tem se tornado cada vez mais importante na prática e que ainda são necessários mais estudos para se consolidarem as informações, bem como auxiliar alguns pacientes que ainda possuem uma qualidade de vida ruim. As limitações do " $n$ " da pesquisa estiveram relacionadas à recusa de alguns dos usuários cadastrados, a dificuldade de estabelecer contato com outros e a situação epidemiológica do mundo que impossibilitou a pesquisa de ser continuada e finalizada presencialmente. Outra dificuldade foi que alguns pacientes não sabiam olhar e informar a FEVE no ecocardiograma.

\section{REFERÊNCIAS}

1. BARBOSA RR, et al. Análise da Qualidade de Vida em Homens e Mulheres Portadores de Insuficiência Cardíaca. Ver Bras cardiol., 2014; 27(2): 97-103.

2. BOISVERT S, et al. An integrative literature review on nursing interventions aimed at increasing self-care among heart failure patients. Rev Latino-Am Enferm., 2015; 23(4): 753-768.

3. CARVALHO VO, et al. Validação da Versão em Português do Minnesota Living with Heart Failure Questionnaire. Arq Bras Cardiol., 2009; 93(1): 39-44.

4. CHIODELLI GC, et al. Relação das forças musculares respiratória e periférica com a limitação nas atividades de vida diária em pacientes com insuficiência cardíaca. Rev. bras. Cien. e Mov., 2015; 23(1): 136-145.

5. FREITAS AKE, et al. Manejo ambulatorial da insuficiência cardíaca crônica. Rev. Med., 2017; 4(3): 123-136.

6. FIGUEIREDO JHC, et al. Efeito Sinérgico da Gravidade da Doença, de Sintomas de Ansiedade e da Idade Avançada sobre a Qualidade de Vida de Pacientes Ambulatoriais com Insuficiência Cardíaca. Arq. Bras. de Cardio., 2019; 114: 25-32.

7. FERNANDES ADF, et al. Insuficiência Cardíaca no Brasil Subdesenvolvido: Análise de Tendência de Dez Anos. Arq. Bras. Cardiol., 2020; 114(2): 222-231.

8. JORGE AJL, et al. avaliação da qualidade de vida em pacientes com e sem insuficiência cardíaca na atenção primária. Arq. Bras. de Cardio., 2017; 109(3): 248-252.

9. KUROGI EM, et al. Relação entre capacidade funcional, desempenho e sintomas em pacientes internados com insuficiência cardíaca. Rev Bras Enferm., 2020; 73(4): e20190123.

10. LIMA PBD, MORAIS ERD. Qualidade de vida e nível de atividade física de pacientes portadores de insuficiência cardíaca crônica. ASSOBRAFIR Ciência, 2014; 5(1): 27-39.

11. MAIA RJC, et al. Strain Longitudinal Global é Preditor de Baixa Capacidade Funcional em Pacientes com Insuficiência Cardíaca Sistólica. Arq. Bras. Cardiol., 2019; 113(2): 188-194.

12. MIZZACI CC, et al. Tratamento farmacológico para insuficiência cardíaca sistólica crônica e as evidências disponíveis: uma revisão narrativa da literatura. Diagn. Tratamento, 2017; 22(1): 8-20.

13. MOURA LZ, et al. Uso de betabloqueadores em pacientes maiores de 65 anos com insuficiência cardíaca sistólica. Rev. Med., 2016; 3(3): 142-147.

14. MURAD RV, et al. Insuficiência cardíaca: o benefício da terapia com betabloqueadores. Rev. Cader. de Med., 2018; (1) $1: 73-77$.

15. NOGUEIRA IDB, et al. Correlação entre Qualidade de Vida e Capacidade Funcional na Insuficiência Cardíaca. Arq. Bras. Cardiol., 2010; 95(2): 238-243.

16. PAZ LFA, et al. Qualidade de vida relacionada à saúde em pacientes com insuficiência cardíaca. Rev Bras Enferm., 2019; 72(2): 148-154. 
17. PELEGRINO VM, et al. Determinantes da qualidade de vida relacionada à saúde em pacientes ambulatoriais com insuficiência cardíaca. Rev. Latino-Am. Enferm., 2011; 19(3): 451-457.

18. ROHDE LEP, et al. Diretriz brasileira de insuficiência cardíaca crônica e aguda. Arquiv. Bras. de card., 2018; 11(3): 436-539.

19. SACCOMANN ICR, et al. Qualidade de vida relacionada à Saúde em Idosos com Insuficiência cardíaca: avaliação com instrumento específico. Acta Paul Enferm., 2011; 24(2): 179-184.

20. SOUSA MMD, et al. Efeitos físicos e psicossociais da insuficiência cardíaca na percepção da qualidade de vida. Cogitare Enferm., 2017; 22(2): 49783.

21. SOUSA MM, et al. Associação das condições sociais e clínicas à qualidade de vida de pacientes com insuficiência cardíaca. Rev Gaúcha Enferm., 2017; 38(2): e65885. 